VOL. $3(1970), 163-170$.

\title{
An orthomodular poset which does not admit a normed orthovaluation
}

\author{
Peter D. Meyer
}

\begin{abstract}
It is of relevance to studies in the logic of quantum mechanics whether or not every separable completely orthomodular poset admits a normed $\sigma$-ortho-valuation. A finite orthomodular poset is constructed which is a counter-example to this proposition.
\end{abstract}

We review some standard notions: An orthocomplemented poset $P$ is an orthoposet if $x \vee y$ exists for any orthogonal $x, y \in P$. An orthocomplemented poset $P$ is a complete orthoposet if every orthogonal subset of $P$ has a least upper bound in $P$. An orthoposet $(P, \leq, \perp)$ is orthomoduzar if for any $x, y \in P, x=y^{\perp}$ if $x \leq y^{\perp}$ and $x \vee y=1$. A complete orthoposet is completely orthomodular if it is orthomodular. A poset $P$ is separable if every orthogonal subset of $P$ is countable (that is, is finite or countably infinite).

Let $(P, \leq, \perp)$ be an orthoposet, then a real-valued function $p$ on $P$ is a normed orthovaluation if:

$$
\begin{aligned}
& \text { (i) } p(x) \geq 0 \text { for all } x \in P \text {; } \\
& \text { (ii) } p(1)=1 \text {, and } \\
& \text { (iii) if } x \neq y \text { and } x \leq y^{\perp} \text { then } p(x \vee y)=p(x)+p(y) \text {. }
\end{aligned}
$$

It can be shown that a normed orthovaluation maps $P$ into $[0,1]$, and in general behaves like a probability function.

Received 14 May 1970. Communicated by P.D. Finch. 163 
Let $(P, \leq, \perp)$ be a separable complete orthoposet, then a normed orthovaluation $p$ on $P$ is a normed o-orthovaluation if (as well as (iii)) $p(v X)=\sum_{x \in X} p(x)$ for any orthogonal subset $X$ of $P$ ( $X$ must be countable since $P$ is separable). The requirement in (iii) that $x \neq y$ is for practical purposes without significance, but is imposed solely so that

(a) the boolean lattice of all subsets of the empty set (a lattice of one element only) behaves itself (as befits its triviality) by admitting a normed orthovaluation, and

(b) the notion of a normed $\sigma$-orthovaluation is (as it is supposed to be) a restriction of the notion of a normed orthovaluation.

In this paper we assume familiarity with Section 1 of Finch [1], which is concerned mainly with the notions of a logical structure and of a logical $\sigma$-structure. A logical structure is a set of boolean lattices with a common 0-element and a common 1-element, satisfying a number of conditions, among which is that the partial orderings, orthocomplementations, and $v$-functions of any two lattices 'coincide' for the elements in their intersection. 'Combining' the boolean lattices in a logical structure produces an orthomodular poset. For the details the original paper should be consulted. If $L=\left\{B_{\gamma}: \gamma \in \Gamma\right\}$ is a logical structure then the partial ordering, the orthocomplementation, and the $v$-function of $B_{\gamma}$ will be denoted by $\leq_{\gamma}, N_{\gamma}$ and $v_{\gamma}$ respectively. Proofs will be terminated by the sign //.

Finch [2] introduces the notion of a state of a physical system associated with a separable logical $\sigma$-structure $L$, and remarks that any normed $\sigma$-orthovaluation on the logic $L$ associated with $L$ ( $L$ is always a separable completely orthomodular poset) determines a state of the physical system (although not all of its states arise in this way). In the concluding section of his paper, Finch raised four questions, one of which is: Does every separable completely orthomodular poset admit at least one normed o-orthovaluation? It is the purpose of this paper to provide a negative answer to this question.

We define a set $L$ of seven boolean lattices as follows: Let 
$B_{1}, B_{2}, B_{3} \in L$ where, for $1 \leq i \leq 3,\left(B_{i}, \leq_{i}, N_{i}\right)$ is the boolean lattice of all subsets of the set $\left\{a_{i}, b_{i}, c_{i}, d_{i}\right\}$. We identify the l-elements of each of these lattices, and denote it by $I$, so that $I$ is the common l-element of $B_{1}, B_{2}$ and $B_{3}$.

Hereinafter $i$ and $j$ will denote arbitrary elements of $\{1,2,3\}$, and $s$ and $t$ will denote arbitrary elements of $\{a, b, c, d\}$. The remaining four boolean lattices making up $L$ are defined as follows: Let

$$
B_{t}=\left\{\square,\left\{t_{1}\right\},\left\{t_{2}\right\},\left\{t_{3}\right\}, N_{1}\left\{t_{1}\right\}, N_{2}\left\{t_{2}\right\}, N_{3}\left\{t_{3}\right\}, I\right\}
$$

(where $\square$ denotes the empty set). The complement of $\left\{t_{i}\right\}, N_{t}\left\{t_{i}\right\}$, is defined to be $N_{i}\left\{t_{i}\right\}$, and the partial ordering $\leq_{t}$ is defined so as to make $\left(B_{t}, \leq_{t}, N_{t}\right)$ a boolean lattice with the following structure:

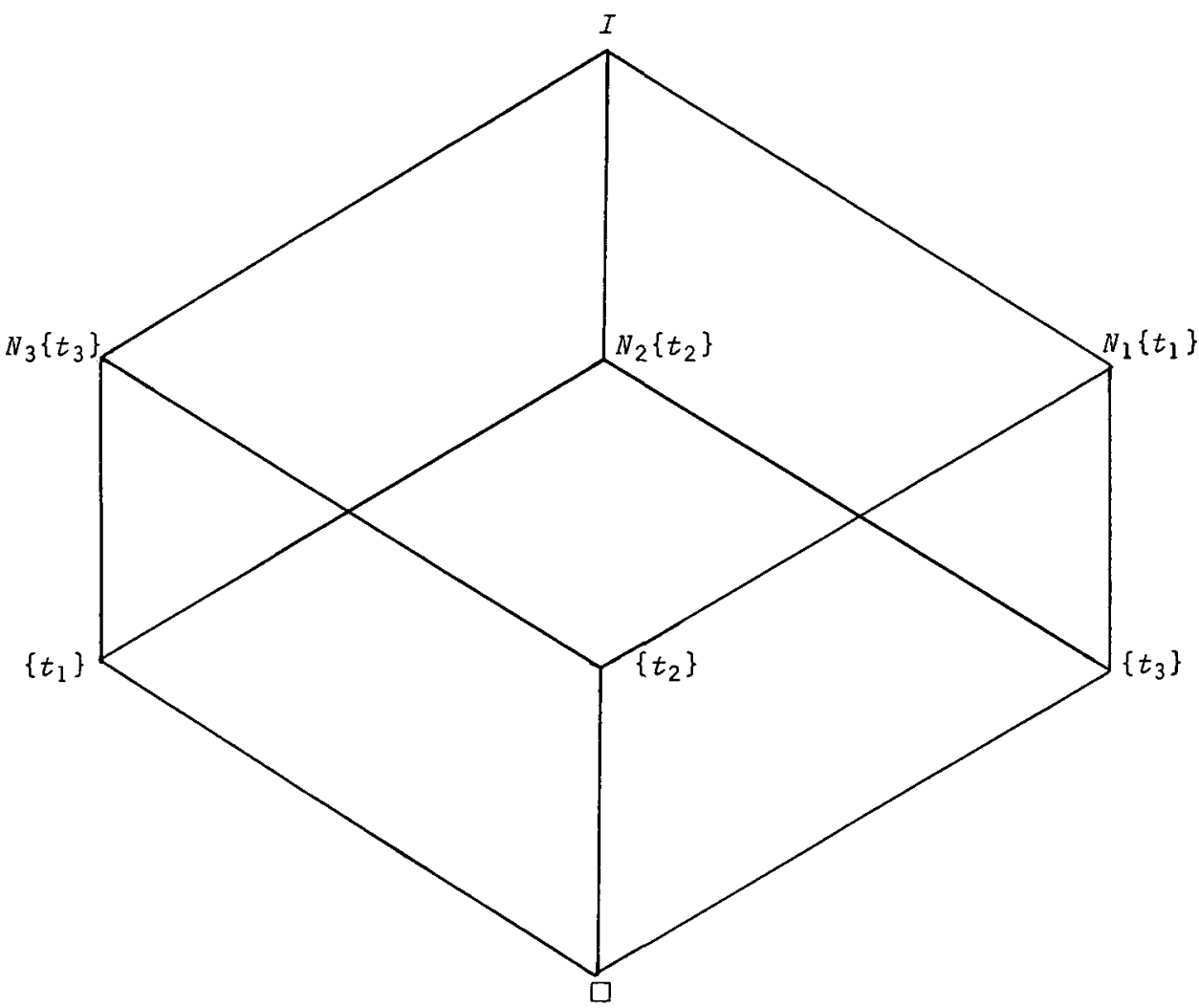


Thus $L=\left\{B_{1}, B_{2}, B_{3}, B_{a}, B_{b}, B_{c}, B_{d}\right\}$ is a set of boolean lattices. Let $B_{3}=\left\{B_{1}, B_{2}, B_{3}\right\}$ and $B_{4}=\left\{B_{a}, B_{b}, B_{c}, B_{d}\right\}$.

LEMMA 1.

(i) If $i \neq j$ then $B_{i} \cap B_{j}=\{\square, I\}$;

(ii) if $s \neq t$ then $B_{s} \cap B_{t}=\{\square, I\}$;

(iii) $B_{i} \cap B_{t}=\left\{\square,\left\{t_{i}\right\}, N_{i}\left\{t_{i}\right\}, I\right\}$.

Proof. Apparent. //

LEMMA 2. L is a logical stmicture.

Proof. We must show that $L$ satisfies conditions (i) - (vi) in the definition of a logical structure given on p. 276 of Finch [1]. In this proof, occurrences of small Roman numerals will correspond to the conditions so numbered in the definition. Let $\left\{B_{\gamma}: \gamma \in \Gamma\right\}$ be an enumeration of $L$.

(i) Each $B_{\gamma}$ has the same O-element, namely, $\square$.

Let $x, y \in B_{\alpha} \cap B_{\beta}$. If $\alpha=\beta$ then:

(1) (ii) $x \leq_{\alpha} y$ if and only if $x \leq_{\beta} y$;

(2) (iv) $N_{\alpha} x=N_{B} x$;

(3) (v) $x v_{\alpha} y=x v_{\beta} y$.

Suppose $\alpha \neq \beta$. If $B_{\alpha}, B_{\beta} \in B_{3}$ or $B_{\alpha}, B_{\beta} \in B_{4}$ then (by Lemma 1) $x, y \in\{\square, I\}$, so (1) - (3) hold.

If $B_{\alpha}=B_{i}$ and $B_{\beta}=B_{t}$ then (by Lemma 1) $x, y \in\left\{\square,\left\{t_{i}\right\}, N_{i}\left\{t_{i}\right\}, I\right\}$, so again (1) - (3) hold.

Similarly if $B_{\alpha}=B_{t}$ and $B_{\beta}=B_{i}$. Hence for any $x, y \in B_{\alpha} \cap B_{\beta}$, (1) - (3) hold.

(iii) Suppose $x \leq_{\alpha} y$ and $y \leq_{\beta} z$. We must show that for some 
$\gamma \in \Gamma, x \leq_{\gamma} z$

If $\alpha=\beta$ then $x \leq_{\alpha} z$. Suppose $\alpha \neq \beta$. If $B_{\alpha}, B_{\beta} \in B_{3}$ or $B_{\alpha}, B_{B} \in B_{4}$ then $x, y, z \in\{\square, I\}$, so $x \leq_{\alpha} z$. If $B_{\alpha}=B_{i}$ and $B_{B}=B_{t}$ then $y \in B_{i} \cap B_{t}=\left\{\square,\left\{t_{i}\right\}, N_{i}\left\{t_{i}\right\}, I\right\}$. If $y \in\left\{\square,\left\{t_{i}\right\}\right\}$ then $x \leq_{B} z$, and if $y \in\left\{N_{i}\left\{t_{i}\right\}, I\right\}$ then $x \leq_{\alpha} z$. Similarly if $B_{\alpha}=B_{t}$ and $B_{\beta}=B_{i}$. Hence for some $\gamma \in \Gamma, x \leq_{\gamma} z$.

(vi) Suppose $y \leq_{\alpha} N_{\alpha} x, x \leq_{\beta} z$, and $y \leq_{\gamma} z$. We must show that for some $\delta \in \Gamma, x, y, z \in B_{\delta}$.

If $\beta=\gamma$ then $x, y, z \in B_{\gamma}$. Suppose $\beta \neq \gamma$. If $B_{\beta}, B_{\gamma} \in B_{3}$ or $B_{\beta}, B_{\gamma} \in B_{4}$ then $z \in\{\square, I\}$ (by Lemma I), so $x, y, z \in B_{\alpha}$.

If $x \in\{\square, I\}$ then $x, y, z \in B_{\gamma}$. Suppose $x \notin\{\square, I\}$. Suppose $B_{B}=B_{i}$ and $B_{\gamma}=B_{t}$ then $z \in\left\{\square,\left\{t_{i}\right\}, N_{i}\left\{t_{i}\right\}, I\right\}$ (by Lemma 1 ). If $z \in\{\square, I\}$ then $x, y, z \in B_{\alpha}$; and if $z=\left\{t_{i}\right\}$ then $x \in\left\{\square,\left\{t_{i}\right\}\right\}$, so $x, y, z \in B_{\gamma}$.

Suppose $z=N_{i}\left\{t_{i}\right\}$, then since $B_{\gamma}=B_{t}$, either $y \in\left\{\square, N_{i}\left\{t_{i}\right\}\right\}$ (in which case $\left.x, y, z \in B_{B}\right)$ or $y \in\left\{\left\{t_{1}\right\},\left\{t_{2}\right\},\left\{t_{3}\right\}\right\} \backslash\left\{\left\{t_{i}\right\}\right\}$. Suppose the latter, then $y=\left\{t_{j}\right\}$ for some $j \neq i$, and so $y \in B_{j}, B_{t}$ only. Now $x \notin\{\square, I\}, x \in B_{\beta}=B_{i}$ and $i \neq j$, so $x \notin B_{j}$. Since $x, y \in B_{\alpha}, x \in B_{j}$ or $x \in B_{t}$. Thus $x \in B_{t}$, so $x \in\left\{\square,\left\{t_{i}\right\}, N_{i}\left\{t_{i}\right\}, I\right\} \quad$ (by Lemma 1$), x \leq_{\beta} z=N_{i}\left\{t_{i}\right\}$, and $x \neq \square$, so $x=N_{i}\left\{t_{i}\right\}=z$. Thus $x, y, z \in B_{\gamma}$.

Similarly if $B_{B}=B_{t}$ and $B_{\gamma}=B_{i}$. Hence for some $\delta \in \Gamma$, $x, y, z \in B_{\delta}$. Since $L$ satisfies the required conditions, $L$ is a logical structure. 
We now define our poset, which will consist of 44 elements. Let $P=B_{1} \cup B_{2} \cup B_{3}$, then $P=U L$. For $x, y \in P$ let $x \leq y$ if and only if for some $\gamma \in \Gamma, x \leq_{\gamma} y$. For $x \in P$ let $x^{\perp}=N_{\gamma} x$ for any $\gamma \in \Gamma$ such that $x \in B_{\gamma}$. In the terminology of Finch [1], $(P, \leq, 1)$ is the logic associated with the logical structure $L$.

PROPOSITION 3. $P$ is an orthomodular poset.

Proof. By the previous lemma, $L$ is a logical structure. $P=U\left\{B_{\gamma}: \gamma \in \Gamma\right\}$ so by the remarks on p. 276 of Finch $[1],(P, \leq, \perp)$ is an orthocomplemented poset. By Theorem 1.1 of the same paper, $(P, \leq, \perp)$ is orthomodular.

Let $S$ be an orthocomplemented poset, then (following Finch [1, p. 280]) a frome of $S$ is a maximal orthogonal subset of $S \backslash\{0\}$.

LEMMA 4. Let $(S, \leq, \perp)$ be an orthoposet, and let $p: S \rightarrow[0,1]$ be a normed orthovaluation on $S$. Then for any finite frome $F$ of $S$, $\sum_{w \in F} p(w)=1$

Proof. By induction on $|F|$. Suppose $|F|=1$, then $F=\{1\}$, so $\sum_{\omega \in F} p(\omega)=p(1)=1$.

Suppose the lemma holds for all n-element frames of $S$ (with $n \geq 1)$. Let $F$ be a frame of $S$ such that $|F|=n+1$. Let $x, y \in F$ such that $x \neq y$. Now $x$ is orthogonal to $y$ and $S$ is an orthoposet, so $x \vee y$ exists in $S$. Let $G=(F \backslash\{x, y\}) \cup\{x \vee y\}$, then $|G|=n$. It is easily shown that $G$ is a frame of $S$.

Since $p$ is a normed orthovaluation on $S, p(x \vee y)=p(x)+p(y)$, so

$$
\begin{aligned}
\sum_{w \in F} p(w) & =\sum_{w \in G} p(w)-p(x \vee y)+p(x)+p(y) \\
& =\sum_{w \in G} p(w) \\
& =1
\end{aligned}
$$

by the inductive hypothesis. 
Thus, by induction, the lemma holds for all finite frames of $S$. //

PROPOSITION 5. $P$ is an orthomodular poset which does not admit a normed orthovaluation.

Proof. $P$ is orthomodular by Proposition 3. The atoms of $P$ are the following 12 unit sets:

$$
\begin{array}{llll}
\left\{a_{1}\right\} & \left\{b_{1}\right\} & \left\{c_{1}\right\} & \left\{d_{1}\right\} \\
\left\{a_{2}\right\} & \left\{b_{2}\right\} & \left\{c_{2}\right\} & \left\{d_{2}\right\} \\
\left\{a_{3}\right\} & \left\{b_{3}\right\} & \left\{c_{3}\right\} & \left\{d_{3}\right\} .
\end{array} .
$$

Let $F_{i}=\left\{\left\{a_{i}\right\},\left\{b_{i}\right\},\left\{c_{i}\right\},\left\{d_{i}\right\}\right\}$, and let $F_{t}=\left\{\left\{t_{1}\right\},\left\{t_{2}\right\},\left\{t_{3}\right\}\right\}$, then clearly each $F_{i}$ and each $F_{t}$ is a frame of $P$.

Suppose now that $P$ admits a normed orthovaluation $P, P$ is an orthoposet, so by the previous lemma,

$$
\sum_{w \in F_{i}} p(w)=1 \text { and } \sum_{w \in F_{t}} p(w)=1 \text {. }
$$

Now the $F_{i}$ are pairwise disjoint, as are the $F_{t}$, so

$$
\left\{\left(p(w): w \in F_{1} \cup F_{2} \cup F_{3}\right\}=\sum_{i=1}^{3} \sum_{w \in F_{i}} p(w)=3\right.
$$

and

$$
\sum\left\{p(w): w \in F_{a} \cup F_{b} \cup F_{c} \cup F_{d}\right\}=\sum_{t=a}^{d} \sum_{w \in F_{t}} p(w)=4
$$

But $F_{1} \cup F_{2} \cup F_{3}=F_{a} \cup F_{b} \cup F_{c} \cup F_{d}$, so if $P$ admits a normed orthovaluation then $3=4$. Hence $P$ does not admit a normed orthovaluation. //

COROLLARY $6 . P$ is a completely orthomodular poset which does not admit a normed o-orthovaluation.

Proof. $P$ is orthomodular by Proposition 3 , so $P$ is an orthoposet. Since $P$ is finite, $P$ is a complete orthoposet, and so $P$ is completely orthomodular. Any normed $\sigma$-orthovaluation on a complete 
orthoposet is a normed orthovaluation, so by Proposition $5, P$ does not admit a normed o-orthovaluation. //

\section{References}

[1] P.D. Finch, "On the structure of quantum logic", J. Symbolic Logic 34 (1969), 275-282.

[2] P.D. Finch, "Quantum mechanical physical quantities as random variables", Nanta Math. (to appear).

Monash University,

Clayton, Victoria. 